\title{
Decreased cerebellar blood flow in postinfectious acute cerebellar ataxia
}

\author{
Shinichiro Nagamitsu, Toyojiro Matsuishi, Masatoshi Ishibashi, Yushiro Yamashita,
} Toshihiro Nishimi, Koutarou Ichikawa, Koichi Yamanishi, Hirohisa Kato

\begin{abstract}
Department of Pediatrics and Child Health, Kurume University School of Medicine, Kurume, Japan

S Nagamitsu

T Matsuishi

Y Yamashita

H Kato

Division of Nuclear Medicine and Department of Radiology, Kurume University School of Medicine, Kurume,

Japan

M Ishibashi
\end{abstract}

Department of Pediatrics, Medical Center for Maternal and Child Health, St Mary's Hospital,

Kurume, Japan

T Nishimi

Department of Pediatrics, Emergency Medical Center, Kitakyushu City Yahata Hospital, Kitakyush, Japan K Ichikawa

Department of Bacteriology, Osaka University, Suita, Japan

K Yamanishi

Correspondence to: Dr Masatoshi Ishibashi, Division of Nuclear Medicine and Department of Radiology, Kurume University School of Medicine, 67 Asahi-Machi, Kurume City, Fukuoka, 830-0011, Japan. Telephone 008194237 2025; fax 0081 94237 2025; email ishi@kurume.ktarn.or.jp

Received 14 July 1998 and in revised form

2 December 1998

Accepted 16 January 1999

\begin{abstract}
Objective-The aim of the present study was to evaluate the regional cerebral blood flow (rCBF) in patients with postinfectious acute cerebellar ataxia using single photon emission computed tomography (SPECT).

Methods-Five children with postinfectious acute cerebellar ataxia and five control subjects were examined. The distribution of rCBF was measured by SPECT imaging after intravenous administration of ${ }^{123} \mathrm{I}-\mathrm{IMP}(111 \mathrm{MBq})$. The $\mathrm{rCBF}$ ratio-defined as the ratio of $\mathrm{rCBF}$ in the region of interest (ROI) to that in the occipital cortex-was calculated for each cortical and subcortical ROI. The mean rCBF ratio of each region was then compared between the ataxic and control subjects. These patients and all control subjects were also evaluated using MRI.

Results-The rCBF ratio was significantly lower in the cerebellum of the ataxic patients than in the cerebellum of the control subjects $(p<0.05)$. No abnormal cerebellar morphology and no abnormal signal intensities were found on MRI.

Conclusion- ${ }^{123}$ I-IMP SPECT clearly demonstrated the decreased $\mathrm{rCBF}$ in the cerebellum of all patients with postinfectious acute cerebellar ataxia.
\end{abstract}

(F Neurol Neurosurg Psychiatry 1999;67:109-112)

Keywords: cerebral blood flow; ${ }^{123}$ I-IMP SPECT; acute cerebellar ataxia

Acute cerebellar ataxia is a relatively common condition in children and is characterised by sudden gait disturbance, truncal ataxia, and nystagmus after infectious illness, usually viral and most commonly varicella. ${ }^{1}$ Although behavioural or learning difficulties after acute cerebellar ataxia occur in about $20 \%$ of such children, ${ }^{2}$ the pathogenesis is unknown. The condition has been postulated to occur as a result of direct viral invasion into the cerebellum or to be an autoimmune response to some agent. ${ }^{3}$ Using MRI or $\mathrm{CT}$, demyelination and inflammatory changes have been reported in patients with this disease. ${ }^{4-6}$ However, the rate of detection of abnormal findings using these brain imaging techniques is very low. Recent advances in neuroimaging, in particular, single photon emission computed tomography (SPECT), have prompted more comprehensive evaluation of cerebral blood flow (CBF) in children with neurological disorders. To our knowledge, brain SPECT findings in patients with acute cerebellar ataxia have not yet been reported.

Patients and methods

PATIENT POPULATION

We evaluated five patients (one boy and four girls, age range 2.0 to 4.0 years, mean 2.3 years) with postinfectious acute cerebellar ataxia. Three had varicella and one had mumps as preceding infections, which were confirmed by a significant rise in virus specific antibody titres in serum. The fifth patient had non-specific virus illness. The diagnosis of postinfectious acute cerebellar ataxia was based on the presence of preceding infection within 3 weeks before the onset of the characteristic symptoms including ataxic gait, nystagumus, and tremor. ${ }^{3}$ No patients had visual problems and all patients recovered from ataxia within 2 weeks. The latent period of four patients was 1 week and that in the fifth patient was 3 weeks. During their stay in hospital, all patients underwent MRI and CSF examination. A CSF specimen from each of the three patients with varicella was analysed for the presence of varicella zoster virus DNA using the polymerase chain reaction (PCR) according to our previously described method. ${ }^{7}$ The SPECT study was performed in each patient within 7 days after the onset of ataxia.

Control subjects were selected a posteriori from a series of 24 children aged 2 months to 16 years who had had $\mathrm{rCBF}$ studies using SPECT in our centre, because they had neurological problems that were found retrospectively to be transient and unaccompanied by cerebral lesions. At the time of the present study, the results of their neurological examinations, EEGs, and CT or MRI studies were normal, and the children remained neurodevelopmentally normal (clinical follow up of 1 year). Of this series, we selected five controls who were age matched to the children with acute cerebellar ataxias. The five control subjects consisted of three boys and two girls, aged range 0.8 to 4.6 years, mean 2.3 years.

BRAIN SPECT

SPECT was performed after injection of; an ${ }^{123} \mathrm{I}$-IMP bolus (111MBq) at rest. Before IMP 


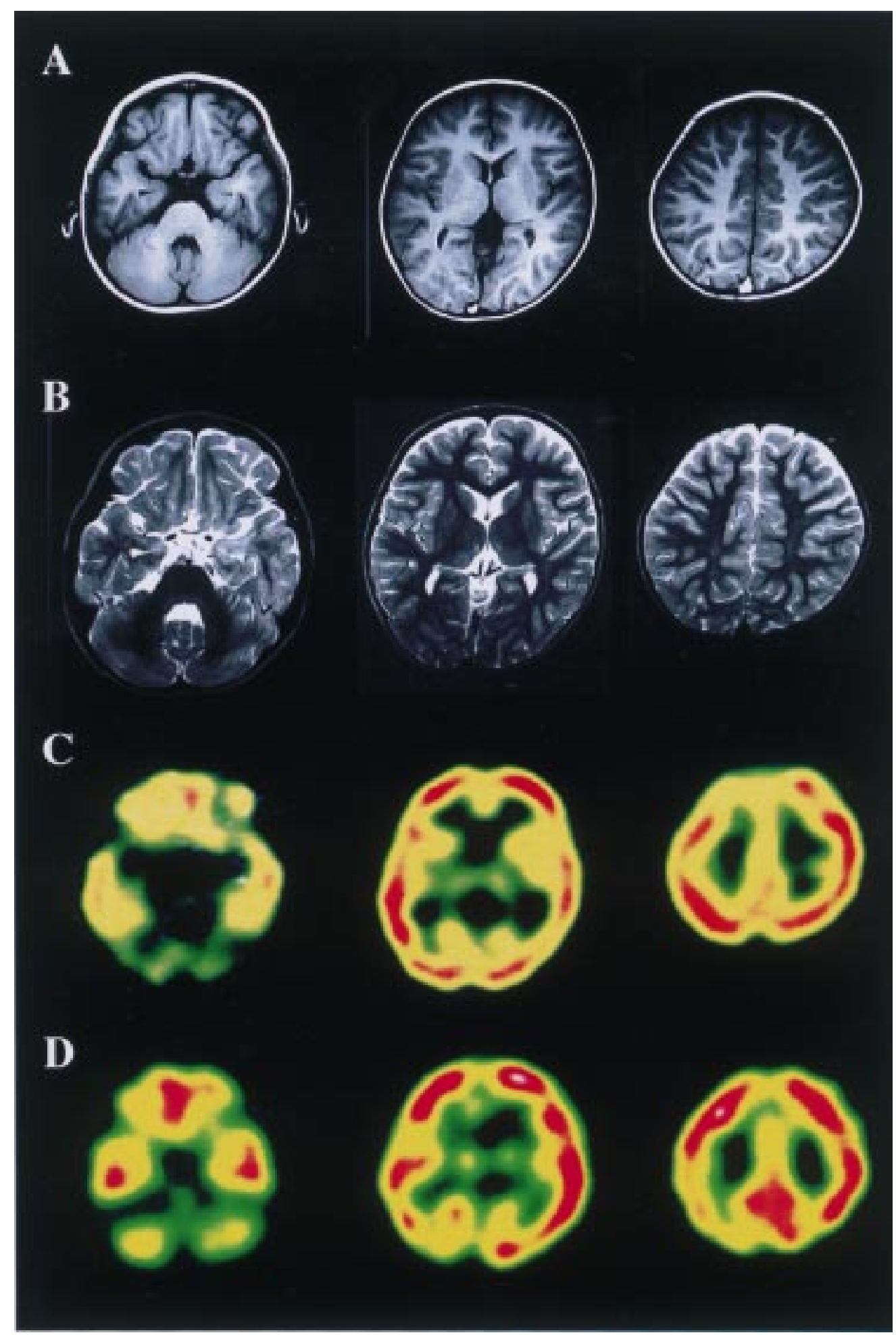

Axial MRI at three different levels in patient 3 with postinfectious acute cerebellar ataxia shows no definite abnormality in (A) T1 weighted and (B) T2 weighted image. (C) ${ }^{223}$ I-IMP SPECT images at the three different levels in patient 3. Decreased regional cerebral blood flow ( $r C B F$ ) was detected in the region of the cerebellum. $(D)^{223} I-I M P S P E C T$ images at the same levels in a control. The cerebral blood flows were normal.

injection, each subject drank a bolus of diluted Lugol's solution to block thyroid uptake of circulating free iodine. Images were acquired using a large field of view, dual detector camera and computer system (RC2600I and RW3000, Hitachi, Tokyo, Japan) equipped with a low energy high resolution parallel hole collimator. SPECT acquisition was initiated about 20 minutes after tracer injection. Pentobarbitone sodium, which was the recommended procedure guideline for paediatric sedation in nuclear medicine, was used after tracer injection in patients with acute cerebellar ataxia and controls.

All the patients' and controls' eyes were closed during the injection of tracer. 
Comparison of the ROI/occipital cortex ratio between subjects and controls

\begin{tabular}{llll}
\hline Regions & $\begin{array}{l}\text { Acute cerebellar ataxia } \\
(n=5)\end{array}$ & Controls $(n=5)$ & $p$ Value \\
\hline Cerebellum & $0.716(0.123)^{\star}$ & $0.879(0.075)$ & 0.035 \\
Temporal & $1.036(0.036)$ & $1.048(0.075)$ & 0.755 \\
Parietal & $1.083(0.071)$ & $1.062(0.078)$ & 0.653 \\
Frontal (sup) & $1.114(0.073)$ & $1.078(0.099)$ & 0.531 \\
Frontal (mid) & $1.061(0.024)$ & $1.023(0.115)$ & 0.490 \\
Striatum & $0.947(0.093)$ & $1.016(0.042)$ & 0.169 \\
Thalamus & $0.908(0.097)$ & $0.925(0.102)$ & 0.794 \\
White matter & $0.790(0.202)$ & $0.767(0.142)$ & 0.840 \\
\hline
\end{tabular}

ROI=the region of interest $(\mathrm{ROI}) ; \mathrm{n}=$ number of patients; sup=superior; mid=middle. * Significantly different from controls $(p<0.05)$.

Continuous transaxial tomograms were reconstructed after back projection was filtered using a Butterworth filter to reduce statistical noise. Subsequently, the cortical and subcortical regions of interest (ROIs) in the acquired SPECT data were defined and the relative blood flow ratio was calculated for each region by dividing the average tissue activity in the region by the activity in the occipital cortex. Because especially the frontal lobe may be subject to significant variations of blood flow depending on the state of consciousness, mood, and alertness, factors which may change rapidly in children between 2 and 4 years of age, the occipital lobe may be ideal as a reference region to build a ratio with the ROIs; ROIs were placed using elliptical templates over the following regions: superior frontal, middle frontal, parietal, temporal, occipital, cerebellum, striatum, thalamus, and white matter regions in each hemisphere. Placement of ROI depended on visual identification of anatomical regions aided by a stereotaxic atlas. ${ }^{9}$ All ROIs were applied by the same observer.

MRI

Brain MRI were acquired on a superconducting magnet operating at $1.5 \mathrm{~T}$ (Magnex $150 \mathrm{HP}$, Shimadzu Co, Kyoto, Japan) using a head coil. The imaging parameters were as follows: conventional spin echo sequences (repetition time (ms)/echo time (ms)) of 500/15 for $\mathrm{T} 1$ weighted images, and $4500 / 80$ for T2 weighted images; section thickness of $5 \mathrm{~mm}$ with $1 \mathrm{~mm}$ interslice gap; $512 \times 512$ acquisition matrix. The $\mathrm{T} 1$ weighted images were also acquired after administration of Gd-DTPA. Two observers with no prior knowledge of the results of the other imaging studies, independently evaluated the MRI for the presence of abnormal areas. Any abnormal appearance on $\mathrm{T} 1$ weighted or T2 weighted images, or any enhancement after administration of $\mathrm{Gd}-$ DTPA, was recorded for analysis as a potential characteristic or identifying abnormality.

INFORMED CONSENT AND INSTITUTIONAL REVIEW OF THE STUDY

Examination of brain imagings and CSF specimens were performed with the written informed consent from the parents of each patient. The procedure for obtaining informed consent and this research received prior approval by the medical ethics committee of Kurume University Hospital.
STATISTICS

The ratios of the $\mathrm{rCBF}$ in the ROI in each region of the cortex to that in the occipital cortex are presented as the mean (SD). The Mann-Whitney test was used to compare mean values in each group. A level of $p<0.05$ was considered to be significant.

\section{Results}

CSF FINDINGS

A lumber puncture was performed in four patients; all showed mild pleocytosis (9-32 cells $/ \mathrm{mm}^{3}$ ). Protein and glucose concentrations in CSF were normal. Varicella zoster viral DNA was not detected in the CSF of the three patients after varicella.

MRI AND SPECT

Brain MRI disclosed no abnormality in structure and signal intensities in the cerebellum of these five patients were normal. The MRI and SPECT images of the representative patient and the control are shown in the figure. The mean $\mathrm{rCBF}$ ratio was significantly lower only in the cerebellum in the patients with acute cerebellar ataxia, compared with the ratio obtained in controls $(p<0.05$, table $)$.

\section{Discussion}

The presence of a latent period from the end of the preceding infection to the onset of ataxia suggests that an autoimmune response to the cerebellum is a more likely aetiological agent than viral invasion to the cerebellum. ${ }^{3}$ In a previous report of this disorder using MRI, a high signal intensity was found in the T2 weighted image, without enhancement in the Gd-DTPA enhanced $\mathrm{T} 1$ weighted image in both the cerebellar cortex and white matter. ${ }^{4-6}$ These findings suggested that an autoimmune response after infectious illness caused demyelination in the cerebellum. However, Connolly et al have reported that the rate of detection of abnormal findings by T2 weighted MRI was very low, only one of 46 cases in this disorder. ${ }^{2}$ Brain MRI in our five patients showed no abnormality, but our SPECT data showed the abnormality of $\mathrm{rCBF}$ in the cerebellum during the acute stage of the disease. To our knowledge, the present study is the first to examine postinfectious acute cerebellar ataxia using brain SPECT. There are no other reports in which ${ }^{123} \mathrm{I}$-IMP has been used to demonstrate the presence of decreased $\mathrm{rCBF}$ in the cerebellum in this disorder.

Postinfectious acute cerebellar ataxia exhibits similar symptoms and form of onset to acute disseminated encephalomyelitis (ADEM). ${ }^{10}$ The symptoms of ADEM appear abruptly within 3 weeks after infectious illness or vaccination and include impairment in consciousness, paresis, cranial nerve signs, and ataxia. However, unlike postinfectious acute cerebellar ataxia, MRI shows abnormal demyelination and oedematous findings in patients with ADEM, and thus this is a useful diagnostic tool. ${ }^{11}$ Abnormal findings in patients with ADEM may also be found using SPECT. ${ }^{12}$ Furthermore, SPECT imaging of some patients with CNS lupus has been shown to be 
more sensitive than MRI. ${ }^{13}$ The detection of abnormal rCBF using SPECT, despite the absence of any abnormality on MRI, has been well demonstrated in some patients with CNS lupus. ${ }^{14}$ This discordant finding between SPECT and MRI suggests that the pathogenesis of CNS lupus may involve decreased CBF such as vasculitis or vasculopathy due to an autoimmune response.

In the present study, all five patients presented clearly characteristic abnormal findings of decreased $\mathrm{rCBF}$ in the cerebellum on SPECT imaging, without abnormal findings on MRI in patients with postinfectious acute cerebellar ataxia.

1 Gieron-Korthals MA, Westberry KR, Emmanuel PJ. Acute childhood ataxia: 10-year experience. $\mathcal{F}$ Child Neurol 1994;9:381-4.

2 Connolly AM, Dodson WE, Prensky AL, et al. Course and outcome of acute cerebellar ataxia. Ann Neurol 1994;35: 673-9.

3 Menkes HJ. Paroxysmal disorders. In: Menkes HJ, ed. Textbook of child neurology. 4th ed. Philadelphia: Lea and Febiger, 1990:620-3.

4 Shoji H, Hirai S, Aramaki M, et al. CT and MR imaging of acute cerebellar ataxia. Neuroradiology 1991;33:360-1.
5 Aufricht CA, Tenner W, Stiglbauer R, et al. Transient cerebellopontine demyelinisation revealed by MRI in acute cerebellar ataxia. Pediatr Radiol 1990;20:602-3.

6 Nakagawa E, Yamanouchi H, Sakuragawa N, et al. Vermis lesions in acute cerebellar ataxia: a sequential imaging study. Brain Dev 1994;16:488-90.

7 Ozaki T, Kajita Y, Asano Y, et al. Detection of varicella zoster virus DNA in blood of children with varicella. $7 \mathrm{Med}$ Virol 1994;44:263-5.

8 Mandel GA, Cooper JA, Majd M, et al. Procedure guideline for patients sedation in nuclear medicine. 7 Nucl Med 1997;38:1640-3.

9 Talairach J, Toumoux P, Rayort M. Co-planar stereotaxic atlas of the human brain. Three-dimensional proportional system: an approach to cerebral imaging. New York, NY: Thieme, 1998: $1-122$.

10 Dyken PR. Viral diseases of the nervous system. In: Swaiman KF, ed. Pediatric neurology principles and practice. 2nd ed. St Louis: Mosby; 1994:669-70.

11 Caldemeyer KS, Smith RR, Harris TM, et al. MRI in acute disseminated encephalomyelitis. Neuroradiology 1994;36: $216-20$

12 Broich K, Horwich D, Alavi A. HMPAO-SPECT and MRI in disseminated encephalomyelitis. F Nucl Med 1991;32: in disseminate

13 Grünwald F, Schomburg A, Badali A, et al. 18FDG PET and acetazolamide-enhanced $99 \mathrm{mTc}$-HMPAO SPECT in systemic lupus erythematosus. Eur f Nucl Med 1995;22: 1073-7.

14 Szer-IS, Miller JH, Rawlings D, et al. Cerebral perfusion abnormalities in children with central nervous system manifestations of lupus detected by single photon emission computed tomography. $\mathcal{F}$ Rheumatol 1993;20:2143-8. 